\title{
FDMine: a graph mining approach to predict and evaluate food-drug interactions
}

\section{Md Mostafizur Rahman}

StFX: Saint Francis Xavier University https://orcid.org/0000-0002-1636-7793

\section{Srinivas Mukund Vadrev}

StFX: Saint Francis Xavier University https://orcid.org/0000-0001-9403-9528

\section{Arturo Magana-Mora}

Saudi Aramco: Saudi Arabian Oil Co https://orcid.org/0000-0001-8696-7068

Jacob Levman

StFX: Saint Francis Xavier University https://orcid.org/0000-0002-9604-3157

Othman Soufan ( $\nabla$ osoufan@stfx.ca)

St. Francis Xavier University https://orcid.org/0000-0002-4410-1853

\section{Research article}

Keywords: Food-Drug Interaction, Link Prediction, Graph Mining, Adverse Effect, Structure Similarity Profile

Posted Date: January 13th, 2022

DOI: https://doi.org/10.21203/rs.3.rs-613772/v2

License: (c) (i) This work is licensed under a Creative Commons Attribution 4.0 International License. Read Full License 


\section{FDMine: a graph mining approach to predict and evaluate food- 2 drug interactions}

3

4 Md. Mostafizur Rahman ${ }^{1}$, Srinivas Mukund Vadrev ${ }^{1}$, Arturo Magana-Mora ${ }^{2}$, Jacob Levman ${ }^{1}$ and 5 Othman Soufan ${ }^{1}$

6

7

\section{Corresponding author}

12 Correspondence to Jacob Levman (jlevman@stfx.ca) or Othman Soufan (osoufan@stfx.ca)

\section{Abstract}

14 Food-drug interactions (FDIs) arise when nutritional dietary consumption regulates biochemical mecha15 16 nisms involved in drug metabolism. Towards characterizing

the nature of food's influence on pharmacological treatment, it is essential to detect all possible FDIs. In this study, we propose FDMine, a novel systematic framework that models the FDI problem as a homogenous graph. In this graph, all nodes representing drug, food and food composition are referenced as chemical structures. This homogenous representation enables us to take advantage of reported drug-drug interactions for accuracy evaluation, especially when accessible ground truth for FDIs is lacking. Our dataset consists of 788 unique approved small molecule drugs with metabolism-related drug-drug interactions (DDIs) and 320 unique food items, composed of 563 unique compounds with 179 health effects. The potential number of interactions is 87,192 and 92,143 when two different versions of the graph referred to as disjoint and joint graphs are considered, respectively. We defined several similarity subnetworks comprising food-drug similarity (FDS), drug-drug similarity (DDS), and food-food similarity (FFS) networks, based on similarity profiles. A unique part of the graph is the encoding of the food composition as a set of nodes and calculating a content contribution score to re-weight the similarity links. To predict new FDI links, we applied the path category-based (path length 2 and 3) and neighborhood-based similarity-based link prediction algorithms. We calculated the precision@top (top 1\%,2\%, and 5\%) of the newly predicted links, the area under the 
31 receiver operating characteristic curve, and precision-recall curve. We have performed three types of eval32 uations to benchmark results using different types of interactions. The shortest path-based method has 33 achieved a precision $84 \%, 60 \%$ and $40 \%$ for the top $1 \%, 2 \%$ and $5 \%$ of FDIs identified, respectively. We 34 validated the top FDIs predicted using FDMine to demonstrate its applicability and we relate therapeutic 35 anti-inflammatory effects of food items informed by FDIs. We hypothesize that the proposed framework 36 can be used to gain new insights on FDIs. FDMine is publicly available to support clinicians and research37 ers.

39 Keywords: Food-Drug Interaction; Link Prediction; Graph Mining; Adverse Effect; Structure Similarity 40 Profile.

\section{Introduction}

42 Drugs bind to targeted receptors on the surface of the cells or enzymes to regulate the rate of chemical reactions. These chemical reactions may be relied upon to treat different diseases and considerably enhance the patients' prognoses. However, drug overdoses or drug interactions may cause critical adverse health conditions. Although the impact of the drugs depends on the affinity of the drug to bind to a specific cell/enzyme receptor, its effectiveness depends on other factors such as when taken alongside other drugs or food. Ideally, drug effects should be consistent across all patients for whom food ingredients do not affect drug response [1]. However, several studies [2,3] have demonstrated the impact of certain foods, decreasing or increasing the activity of different drugs (food-drug interactions - FDI).

FDIs often cause changes in drug plasma concentrations, which may significantly increase or decrease the effectiveness of the drug [4]. These alterations can occur in three ways: they can boost the actions of of drugs (i.e., increased metabolism), reduce the actions of drugs (i.e., decreased bioavailability), or create an adverse effect.

FDIs can be classified into two primary mechanisms: pharmacokinetic (PK) interactions, and pharmacodynamic (PD) interactions [5]. PK interactions denote the circumstance when foods alter processes related to absorption, distribution, metabolism, and excretion of medications. For example, for a short time after consumption, grapefruit juice slows the metabolism of cyclosporine (e.g.: cytochrome P450 enzymes) [6, 7]. PD interactions are caused by specific interactions between a drug and a food component that results in a a pharmacological effect [8]. An example of a PD interaction is a diet high in vitamin $\mathrm{K}$ that antagonizes the therapeutic effects of warfarin (used for blood clot treatments) [5]. 
61 FDIs can affect drug absorption levels and, therefore, are known to impact drug discovery [9].

62 For example, Moringa oleifera leaf extract has been used to inhibit cancer

63 cells and to increase the efficacy of chemotherapy in humans [10,11, 12]. The roots of Erythroxylum per-

64 villei provide pervilleines A, B, C, and F, effective inhibitors of P-glycoprotein, which is linked to multi-

65 drug resistance and low cancer therapeutic response [13]. These are only a few examples that demonstrate

66 the importance of understanding the interactions of food constituents and dietary supplements (containing

67 different chemicals and phytochemicals) with drugs. Consequently, understanding FDIs has the potential 68 to assist physicians, researchers, and patients in reducing ad-verse drug events (ADEs).

69

Earlier research has been mostly based on clinical studies or literature reviews that focus on specific drug interactions with a limited set of foods $[5,8,14,15]$. Based on analyzing PD or PK alterations, these studies examine how food items can affect the efficacy of specific drugs. Some studies have focused on a particular group of patients and examined FDI interactions with the types and number of drugs used (e.g., drugs used for chemotherapy, drugs used as anticoagulants) [16, 17, 18]. Although these studies provided valuable information to physicians about the potentialities of FDIs, the level of novel exploration is limited. Computational approaches can, therefore, potentially be used to predict novel FDIs.

Cheminformatics studies have achieved outstanding results in the fields of drug-drug interactions (DDIs), drug-target interactions (DTIs), and new drug discovery. Multiple computational models have been developed for detecting how a particular drug pair interacts towards new drug discovery.

The adoption of different machine learning models is rapidly increasing in drug discovery [19]. These models have been used for finding new DDIs. For example, Lee et al. Proposed a deep learning model to predict the pharmacological effects of DDIs using structural similarity profile (SSP), target gene similarity profiles, and gene ontology (GO) term similarity profiles of known drug pairs [20]. Recently DeepDDI, was developed as a multi-label classification model that calculates structural similarity profiles (SSP) of DDIs and uses principal components analysis to reduce features and feed them into a feed-forward deep neural network (DNN) [21]. Another predictive model [22] was developed to delineate currently unknown biological effects of inactive ingredients and generally recognized as safe compounds present in food. A general-purpose method, named Alternative Drug-Drug Interaction, was developed to predict the DDIs [23]. Three combined methods were used, including deep learning, text mining, and graph clustering. Feng et al. proposed DPDDI to predict DDIs without considering the biolog-

91 ical and chemical properties [24]. The authors used graph convolution networks (GCN) and DNN as a 
92 predictor. By identifying the topological association of drugs in the DDI network, GCN explores low-di93 mensional feature representations of drugs.

94 Several chemoinformatics studies have successfully demonstrated the application of computational mod95 els for predicting DTIs. Yo et al. [25] used a deep learning model to predict DTIs using a network repre96 sentation. The solution is a linear classification model based on using the least absolute shrinkage and se97 lection operator (LASSO) and LASSO-DNN. LASSO helped in feature extraction to predict DTIs. In one 98 of our previous works, we developed DASPfind [26], a novel computational method to predict the DTIs 99 that uses a simple path (up to 3 lengths) to infer novel drug-protein interactions from a graph structure. The 100 graph was derived from similarities among drug-drug, protein-protein, and known drug-protein interac101 tions. Olayan et al. [27] developed the DDR method for predicting DTIs. The authors constructed a heter102 ogenous graph from the known DTIs and multiple similarities among the drug-drug and target-target inter103 actions, used for feature engineering. The engineered features were later used as inputs for a random forest 104 method to predict the novel DTIs. Different studies have developed link prediction approaches to predict 105 DTIs. Lu et al. [28] used link prediction based on similarity indices to predict DTIs. Fokoue et al. [29] 106 developed the Tiresias framework that uses a large-scale similarity-based link prediction based on different 107 drug data to determine the DDIs. The framework uses a large-scale logistic regression model to predict 108 potential DDIs.

109 Although the implementation has made significant advances of these chemoinformatics models for DDIs 110 and DTIs, FDIs remain poorly addressed. This is mainly due to the inadequacy of resources regarding FDIs 111 since it is often difficult to extract a sufficient number of curated interactions. In addition, for FDI there is 112 no gold standard dataset yet for evaluation. Recently, FooDB [30, 31] was developed as a well-structured 113 and annotated database listing food items and compound composition. Although there is no gold standard 114 dataset for evaluation as in the field of DTIs, we propose using known DDIs. Given the homogenous nature 115 of our graph representations (i.e., all nodes are chemicals), we can resort to certain subnetworks for evalu116 ation. To the best of our knowledge, this is the first work on developing a homogenous graph mining frame117 work for food-drug interactions.

118 In this study, we propose FDMine, a framework that analyzes FooDB [30, 31] and DrugBank [32] data119 bases to create a comprehensive dataset of small molecules with known food-food interactions (FFIs), 120 DDIs, and FDIs. FDMine uses the simplified molecular-input-line-entry system (SMILE) description to 121 establish similarity profiles and link prediction algorithms to predict the FDIs. The proposed framework 122 uses two different kinds of representations (disjoint and joint) graphs consisting of three subnetworks con123 nected. These subnetworks are drug-drug similarity, food-drug similarity, and food-food similarity. The 
124 rationale behind this approach is to capitalize on the similarity information of different subnetworks and

125 combine it with building a homogenous graph. We consider a unique representation of food items, their

126 compound composition, and the contribution of each compound. After building the graph network, the

127 framework implements a comprehensive set of different link prediction algorithms to predict potential

128 FDIs. The shortest path-based method has achieved a precision $84 \%, 60 \%$ and $40 \%$ for the top $1 \%, 2 \%$ and

$1295 \%$, respectively. In the joint version of the graph, FDMine recovered 27,448 links on average from 27,612

130 available (i.e., $99.4 \%$ recovery with standard deviation of $5.1 \mathrm{e}^{\wedge}-4$ ).

\section{Methods}

\section{Databases and datasets preparation}

\section{DrugBank}

134 We used the DrugBank (v 5.1.7) database that contains detailed information for each drug (i.e., chemical, 135 pharmaceutical, and pharmacological data) with extensive drug target information (i.e., sequence, pathway, 136 and structure) [32, 33, 34]. The database contains information for a total of 13,680 different drugs. In Drug137 Bank, drugs are grouped into five categories, including approved, experimental, investigational, nutraceu138 tical, and withdrawn. Drugs can be differentiated as small molecules or biotechnology-driven. The database 139 provides access to the SMILE strings of the drug molecules and reports drug-drug interactions [33].

140 In this study, we considered the drugs assigned to the approved drug group and have small molecules.

141 This resulted in 1,683 drugs. We further reduced this set of molecules by considering only those having

142 "metabolism (increase or decrease)" related interactions, resulting in 788 unique approved small molecule 143 drugs. FDI interactions are mainly detected in relation to metabolic mechanisms [14]. The details of the 144 drug extraction procedure from the DrugBank dataset can be found in the Additional file 1: Fig. S1.

\section{FooDB}

146 We used the FooDB Version 1.0 dataset in JSON format [30, 31], containing several datasets related to 147 foods, compounds, nutrients, and health effects. In this study, we considered the FooDB content dataset 148 that directly mapped foods to the chemical compounds' composition. Initially, we created a subset of the 149 content dataset that stored the required attributes (i.e., food id, original food name, source id, source type, 150 among others), yielding a total of 19,867 objects. Then, we filtered the extracted data by removing the list 151 of predicted and unknown data entries by using the conditions "citation type == DATABASE" and "source 152 type == COMPOUND". This provides a more accurate source of information. Finally, we only considered 153 the food items mapped to a compound, resulting in 16,230 objects for further analysis. 
154 After the parsing step, we mapped the resulting dataset with the "Compound" information to collect the 155 required details for each compound, including SMILE description and content contribution. In FoodDB, 156 the content range of each compound within a food item is presented (e.g., Strawberry has a content range 157 of Potassium of $0.000-187.000 \mathrm{mg} / 100 \mathrm{~g}$ ). Finally, we have the SMILE description of the corresponding 158 compounds and the international chemical key (InChiKey) as a unique identifier.

159 To relate the food compounds to health effects, we retrieved data from the health effects dataset that enabled 160 us to know which food compound has a health effect on the human body. The resulting dataset contains 1618,846 objects including 320 unique foods, and 563 unique food compounds having 179 unique health ef162 fects. One extracted example is that benzoic acid from American cranberry has an allergenic health effect.

163 Since the same compounds can be found in different foods, it is necessary to store these data with a naming 164 convention that allows us to differentiate each food with its composition correctly. In this study, we used 165 the following naming convention: FOODXXXX_FDBXXXXX_CompoundName. For example, the data 166 entries "FOOD00005_FDB000633_Kaempferol" and "FOOD00008_FDB000633_Kaempferol" refer to 167 the same compound Kaempferol with the compound identifier FDB000633 from two different foods 168 (FOOD00005 and FOOD00008). Each compound can be treated differently based on the reported content 169 range in the food item.

170 The data-flow diagram of the extraction procedure of the FooDB dataset can be found in the Additional file 171 1: Fig. S2.

\section{Food composition and compound contribution}

173 Each food item is composed of a set of chemical compounds. Clearly, the "amount of the original content" 174 of any compound is not the same for each food. For example, the amount of the phytic acid in carrot is $1755270.000 \mathrm{ml} / 100 \mathrm{~g}$ and buckwheat is $1800.000 \mathrm{ml} / 100 \mathrm{~g}$. Carrot contains approximately three times more 176 phytic acid than buckwheat by mass. Therefore, the contribution of the phytic acid is different for carrot 177 and buckwheat. Consequently, we used the following equation to calculate the contribution of each com178 pound for each food based on the amount contained in the food:

$$
\text { Contributionscore }(\text { normalized })=\frac{\text { Compoundoriginalcontent } \in \text { afooditem }}{\text { Totaloriginalcontentofallcompounds } \in \text { afood }}
$$

179

180 The range of the normalized contribution is from 0 to 1 . Where 0 and 1 contribution refer to a food com181 pound with no contribution or full contribution, respectively. 
182 In the graph, the food item and its compound composition are represented as separate nodes. The normal-

183 ized contribution score scales edge weights of links connecting compounds to the food item.

185 More details and an example on the contribution score of food compounds is given in the Additional file 1:

186 Table S1.

\section{Homogenous Graph Representation}

188 We consider a set of food compounds, $F=\left\{f_{1}, f_{2}, \ldots, f_{m}\right\}$ and a set of drugs, $D=\left\{d_{1}, d_{2}, \ldots, d_{n}\right\}$ where $189 m$ represents the number of food compounds and $n$ represents the number of drugs. We merged all drugs 190 and food compounds into a single graph. So, in our representation, we have a set of drug and food com191 pounds $F D=\left\{f_{1}, f_{2}, \ldots, f_{m}, d_{1}, d_{2}, \ldots, d_{n}\right\}$. Then, we considered the set of an $m * n$ dimensional struc192 ture similarity matrices between drugs, between food compounds, and between food-drug. A score between $193[0,1]$ is the degree of similarity. A similarity score close to 0 means that two items are not identical to each 194 other, where the most similar items are represented by a similarity score close to 1 . Using this similarity 195 concept, we derived a homogenous graph. From this homogenous graph, we will apply different path cate196 gory and neighborhood-based similarity-based algorithms to predict the novel FDIs.

\section{Structure Similarity Profile}

198 A structural similarity profile (SSP) is a feature vector that contains a unique numerical representation after 199 acquiring structural features of individual food compounds and drugs. The SSP contains pairwise structural 200 similarity scores obtained from the comparison among all the 788 approved small molecule drugs of Drug201 Bank and 8,846 unique food compounds. Structural similarity between a pair of nodes (i.e., drug-drug, 202 food-food, and food-drug) was measured by the Tanimoto coefficient. This coefficient is an efficient way 203 to calculate the structure similarity based on the chemical fingerprint $[35,36]$. The Tanimoto coefficient is 204 defined as the number of common chemical fingerprints compared to the number of all chemical finger205 prints of the two drugs. Chemical fingerprints of each drug were calculated using Morgan/Circular finger206 prints [37] (also known as extended-connectivity fingerprint ECFP4 [38]) that is widely used in different 207 studies. ECFP4 showed the best performing fingerprints in the target prediction benchmarks [39, 40] and 208 in small molecule virtual screening [41]. The calculating procedure of the SSP can be found in the Addi209 tional file 1: Fig S3. 


\section{Sparse Matrix Representation}

211 We used the similarity profile to derive the sparse matrix representation, later used for plotting the graphs.

212 In this matrix, we eliminated all the zero entries and applied a threshold since some similarity scores contain

213 trivial values and thus may not indicate significant changes. For determining the threshold, we have con-

214 sidered the distribution of the similarity scores. The majority of similarity values lie between $0.3 \sim 0.6$, hence

215 selecting a high similarity value may drastically change the data-set size. Also, of note, a high threshold

216 will always lead to potential pairs having increased probability of interaction. Several studies have referred

217 to different values in the range of 0.5-0.85 for applying a similarity threshold for the Tanimoto coefficient

218 [42, 43, 44]. While a higher threshold can lead to more potentially valuable hypotheses, it can limit the

219 number of genuinely novel predictions. Table 1 highlights the number of links of each subnetwork after

220 applying a range of similarity thresholds. Compared to a threshold of 0.6 , a value of 0.7 would result in

$22175 \%$ fewer number of possible FDIs. Therefore, we choose 0.6 at this step. It should be noted that this

222 parameter is provided as an input argument for the user of FDMine.

223

224

Table 1 Number of links in the graph after applying different Tanimoto similarity thresholds

\begin{tabular}{|c|c|c|c|c|}
\hline Tanimoto Threshold & Total Links & DD Links & FF Links & FD Links \\
\hline$>=0.5$ & $5,392,354$ & 14,298 & $5,228,607$ & 149,449 \\
\hline$>=0.6$ & $4,177,383$ & 2,926 & $4,167,202$ & 7,255 \\
\hline$>=0.7$ & $3,834,135$ & 920 & $3,831,336$ & 1,879 \\
\hline
\end{tabular}

225

226

227

\section{Updating Similarity Scores using Food-Compound Contribution}

We obtained a total of 4,177,383 similarities using the SSP. Then, we multiplied the similarity score by the normalized contribution of the food compound (Eq. 1). As illustrated in Table 2, when we have a food-drug pair (see row 1), we multiplied the similarity score by the contribution of the food compound. Similarly, we multiplied the similarity score by the higher contribution of the food compound. For example, the contribution of the FOOD00006_FDB000474_L-Lysine is 0.007301117, and the FOOD00006_FDB000556_ L-Alanine is 0.009780473 . So, we have considered the maximum value of 0.009780473 to update the similarity score. For drug pairs, similarity scores were preserved.

235

$$
\text { Score }=\text { PriorScore }(S S P) * \text { ContributionofFoodCompound }
$$

236 


\begin{tabular}{|c|c|c|c|c|}
\hline nodeA & nodeB & Prior Score & $\begin{array}{c}\text { Contribution } \\
\text { (0 1) }\end{array}$ & New Score \\
\hline DB00136 & FOOD00165_FDB012362_ \\
& beta-Sitosterol & 0.6947674 & 0.3459079 & 0.2403255323224 \\
\hline $\begin{array}{c}\text { FOOD00006_ } \\
\text { FD000474_L- } \\
\text { Lysine }\end{array}$ & $\begin{array}{c}\text { FOOD00006_FDB000556_L- } \\
\text { Alanine }\end{array}$ & 0.6 & 0.009780473 & 0.005868284 \\
\hline
\end{tabular}

238

239 After updating the similarity scores in the graph, we consider another threshold using the contribution score.

240 Here, we consider a more relaxed range $(0.3,0.4,0.5$ and 0.6$)$ as compared to the Tanimoto coefficient

241 threshold. In our literature validation, we prepare and discuss another batch of results using a similarity

242 score of 0.3 , though a value of 0.5 has been employed for the generation of our primary findings. For a

243 threshold of 0.5, we ended up with 87,192 interactions and 92,143 for disjoint and joint dataset respectively.

244 Table S2 in Additional file 1 lists the number of interactions for the considered range.

245 Link Prediction Algorithms

246 After applying the similarity thresholds, the generated graph had several disjoint subgraphs. We call this

247 the disjoint version. Some link prediction algorithms cannot handle the disjoint version. Therefore, we con-

248 sidered preparing a joint graph. We chose any node (randomly) from each subgraph and added an edge to

249 link all subgraphs to make the joint graph network. Then, a very small edge weight of 1e-5 was assigned to

250 the newly added links, limiting their effect on generating biased hypotheses. We generated results for both

251 versions. A detailed description is available in the Additional file 1: Fig S4.

\section{Path Category-based Algorithm}

253 Our goal is to predict the novel (unknown) FDIs from the generated homogenous graph. A homogenous 254 graph is one where all nodes are of the same type. Different than DTI heterogenous graphs (e.g., drug255 protein), nodes in our graph are chemicals. One class of algorithms is based on running the shortest path to 256 find candidate interactions for the considered food and drug pair. Here, we have used 2-length and 3-length 257 pathways. For example, a 2-length path is "Drug1-Food1-Food2" (see Figure 1) connects the Drug1 node 258 with the Food2 node through the similarity between "Drug1 and Food1" and "Food1 and Food2". This is 259 defined as a D-F-F path. As illustrated in Figure 1, the gold color circle denotes the food node and silver 260 color circle denotes the drug node. There are 8 possible combinations of paths (i.e., Drug-Drug-Drug, Drug261 Food-Drug, Food-Food-Food, Food-Drug-Food, Drug-Drug-Food, Drug-Food-Food, Food-Drug-Food, 262 and Food-Food-Food). 
264

265

266

267

268

269

270

271

272

273

274

275

276

277

278

279

280

281

282

283

284

285

286

287

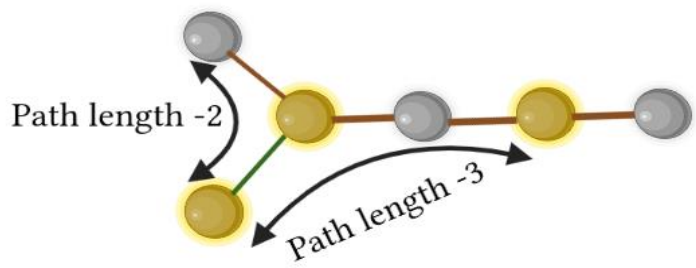

Figure 1 Example working procedure of the Path Category-based method

For predicting new interactions, any path can be followed. The same applies for 3-length pathway prediction. For example, we can get another new link using 3-path length (Food-Food-Drug-Food). The score for the newly predicted link is calculated according to equation 3 , where, $p$ is the path, $n$ is the total number of path and $w$ is the weight of the path:

$$
\text { score }=\operatorname{Min} \sum_{p=1}^{n} P_{w}
$$

Dijkstra's algorithm was used for finding the shortest path where the similarity score is used as the path weight.

\section{Neighbourhood-based Similarity-based Link Prediction}

In the link prediction, given a graph $G$, the main aim is to predict new edges (drug-food) from the existing graph. Predictions are useful to suggest unknown relations (or interactions) based on edges in the observed graph. In the link prediction, we try to build a similarity measure between pairs of nodes and link the most similar nodes. Link prediction algorithms are very common in many application domains such as, identifying protein-protein interactions [45], drug-drug interactions [29], DTIs [28], social networks [46], reconstructing networks [47], document recommendation, recommendation systems [48], biological networks [49], disease prediction [50], bipartite networks [51], etc.

Here, we applied six different types of link prediction algorithm. They are, Adamic and Adar Coefficient (AA) [50, 52], Common Neighbor (CN) [28, 50, 53], Jaccard Coefficient (JAC) [28, 50, 54], Resource Allocation (RA) [50, 55, 56], Multiple Paths of Length L=3 (L3) [45, 57], and Dice Coefficient (Dice) [58, 59]. All of these algorithms have their scoring function. Each of these algorithms assigns a score for the new predicted links. 


\section{Adamic and Adar Coefficient (AA)}

290 The Adamic and Adar Coefficient (AA) gives preference to node pairs with more common neighbors but 291 with a lower degree. If there are no common neighbors for a node pair, then the AA score is 0 . The AA 292 measure is formulated to connect node pairs that have common neighbors.

$$
S_{A A}(a, b)=\sum_{z \in \Gamma(a) \cap \Gamma(b)} \frac{1}{\log k_{z}}
$$

293

294 Here, $a$ and $b$ are two nodes, and $z$ denotes a common neighbor to both $a$ and $b . k$ is the degree of node $295 z$.

296 Common Neighbor (CN)

297 In the Common Neighbor (CN) algorithm, the score for link prediction is computed by finding the number 298 ofcommon neighbors between two distinct nodes. Where, $a$ and $b$ are two nodes. $\Gamma(a)$ and $\Gamma(b)$ denote the 299 set of neighbors of nodes $a$ and $b$, respectively.

$$
S_{C N}(a, b)=|\Gamma(a) \cap \Gamma(b)|
$$

300

301

302

303

304

305

306

307

308

309

310 Resource Allocation (RA) calculates the score based on irregular nodes connecting node $a$ and $b$. The

311 number of resources node $a$ receives from node $b$ through indirect links is called the similarity index. In 
312 the RA each intermediate link contributes a unit of the resource. The RA is also symmetric. $z$ denotes

313 common neighbor of both $a$ and $b$ nodes and k-denotes the degree of node $z$.

$$
S_{R A I}(a, b)=\sum_{z \in \Gamma(a) \cap \Gamma(b)} \frac{1}{k_{z}}
$$

314

\section{Multiple Paths of Length $L=3$ (L3)}

316 Links of high degree nodes prompt multiple and unspecific shortcuts in the network, resulting in biased 317 predictions. This can be avoided by using proper degree of normalization. Such degree of normalization is 318 very important for L3. To eliminate potential degree biases caused by lower degree nodes, we assign a 319 degree normalized L3 score to each node pair $a$ and $b$. Here, $\mathrm{u}$ and $\mathrm{v}$ are intermediate nodes in the 3-length 320 path.

$$
L 3_{a b}=\sum_{u, v \in L 3} \frac{A_{a u} A_{u v} A_{v b}}{\sqrt{k_{u} k_{v}}}
$$

321

\section{Dice Coefficient}

323 Dice coefficient is similar to the Jaccard Coefficient (JAC). The Dice coefficient is calculated using equa324 tion 9, where, $a$ and $b$ are two nodes.

$$
S_{\text {Dice }}(a, b)=\frac{2 *|a \bigcap b|}{|a \cup b|}
$$

\section{Performance evaluation}

327 To measure the performance of applied link prediction approaches, we adopted the idea of precision@k $328[60,61]$ or top $k$ predictive rate $[53,62]$. This metric is also known as $r$-precision $[63,64,65,66]$. preci329 sion@ $\mathrm{k}$ is the recommended measure for link prediction algorithms [67]. It refers to the percentage of true 330 positives among only the top $k$ ranked predicted links. Given the ranked output of the graph, we need to 331 evaluate the ranking precision of the methods.

332 Following [26], we chose the top 1\%,2\%, and 5\% as the value of $k$. In general, the area under the receiver 333 operating characteristic curve (AUROC) or (AUC) is used to evaluate performance of classification models. 334 Nevertheless, recent studies have shown that AUROC is unsuitable for checking the performance of the 335 link prediction algorithms $[56,68,69,70]$. Another statistical measure is the area under the precision-recall 
curve (PRC), which provides a more accurate assessment especially when dealing with imbalanced datasets

337 [71]. In this study, we used, precision@top, AUC, and PRC as performance metrics.

In order to compute some of the measures, we had to derive true positives (TP), false positives (FP), true negatives $(\mathrm{TN})$, and false negatives $(\mathrm{FN})$. To perform this, we ranked the predicted links in descending order based on the rank score given by the link prediction methods. Then, we considered several thresholds as cutoff values. The starting threshold is the minimum score given by the link prediction methods. Then we increase by a step size of 0.1 , which was selected to ensure sufficient granularity in computing the area under the curve. We repeated this step until the threshold value is the same as the maximum score given by the link prediction algorithm. For each specific threshold score, if we found the known link in the test dataset matched with the newly predicted link and the score is greater than the threshold, we considered this matching as a true positive (TP) for evaluative purposes. Given an unknown link, which does not match the test dataset, but was predicted by the link prediction algorithm, and the score is greater than the threshold, we consider the case a false positive (FP). Similarly, when we found a known link (same as the test dataset and in the newly predicted links), but the score was below the threshold, we consider this a false negative (FN). Lastly, when we found any unknown link with the score below the threshold, we assign the sample as a true negative (TN). Using the TP, FP, TN, and FN we calculated the "precision@top-1\%", “precision@top-2\%”, “precision@top-5\%”, AUC, and PRC.

\section{Data splitting for testing}

To evaluating the performance of link prediction algorithms, the test data is generated by excluding a collection of links from the full homogenous networks. Our homogenous network contains drug-drug similarity, food-drug similarity, and food-food similarity. We split $30 \%$ of links randomly to make the test data set, while the rest of the $70 \%$ of links are used for the training dataset. For stability, we repeat this evaluation ten times and report average performance.

\section{Ground-truth evaluation using DDS}

363 Contrary to food-protein interactions [26], there is no accessible gold standard for widely confirmed food364 drug interactions. Therefore, we resorted to the extracted drug-drug interactions from DrugBank for ground 365 truth evaluation. Since the graph representation in FDMine is homogenous (i.e., all nodes are chemicals), 366 we can consider any part of the graph as a representative set of evaluation. Here, we remove $30 \%$ of the 367 drug-drug links in the graph. Then, we execute the framework and report top ranked cases for the precision 
evaluation. We split 30\% DDS links (randomly) for making the test data set, while the rest of the 70\% DDS, and all FDS, FFS links are used in the training dataset. Here, we measured the precision in terms of recovering the original links in the DDS subgraph. It should be noted that we also performed evaluation using a random subset of any type of links (see Results).

372

373 We have performed three types of evaluations to benchmark the results. In the first evaluation, a drug can

374 have a link with another drug because of certain similarity scores. In the second evaluation, a drug will have 375 a correct link with another drug only if it is reported in the DrugBank database. The difference between the 376 second and third evaluation is that the original links in the second evaluation are assumed based on the 377 established similarity measures. Both evaluations will help us establish a comprehensive overview of link 378 recovery in general and the validity of these recovered links using DrugBank. Although drug-drug interactions are examined in these two evaluations, they both provide estimates for the accuracy of food-drug predictions since the graph is homogenous in nature. The following Table 3 lists all the evaluative approaches we have performed in this study.

382

Table 3 List of evaluation approaches

\begin{tabular}{|l|l|l|l|l|}
\hline \multicolumn{1}{|c|}{ Title } & \multicolumn{1}{|c|}{ Evaluation } & \multicolumn{1}{c|}{ Graph } & $\begin{array}{l}\text { Correct predic- } \\
\text { tions }\end{array}$ & \multicolumn{1}{c|}{ Methods } \\
\hline $\begin{array}{l}\text { Evalua- } \\
\text { tion 1 }\end{array}$ & $\begin{array}{l}\text { Remove random 30\% of } \\
\text { links from the DDIs (repeat } \\
10 \text { times) }\end{array}$ & $\begin{array}{l}\text { Comprehensive } \\
\text { evaluation for re- } \\
\text { covery of DDS } \\
\text { similarity links }\end{array}$ & $\begin{array}{l}\text { Match predicted } \\
\text { links with the ac- } \\
\text { tual ones }\end{array}$ & $\begin{array}{l}\text { All methods } \\
\text { are applied }\end{array}$ \\
\hline $\begin{array}{l}\text { Evalua- } \\
\text { tion 2 }\end{array}$ & $\begin{array}{l}\text { Remove random 30\% of } \\
\text { links (repeat 10 times) }\end{array}$ & $\begin{array}{l}\text { Ground Truth us- } \\
\text { ing DrugBank }\end{array}$ & $\begin{array}{l}\text { Match predicted } \\
\text { links with Drug- } \\
\text { Bank reported in- } \\
\text { teractions }\end{array}$ & $\begin{array}{l}\text { SP_the best } \\
\text { trom evalua- } \\
\text { tion 1 over dis- } \\
\text { joint graph) } \\
\text { and RA (the } \\
\text { best from eval- } \\
\text { uation 1 over } \\
\text { joint graph) }\end{array}$ \\
\hline
\end{tabular}




\begin{tabular}{|l|l|l|l|l|l|}
\hline $\begin{array}{l}\text { Evalua- } \\
\text { tion } 3\end{array}$ & $\begin{array}{l}\text { Remove random 30\% of } \\
\text { links (repeat 10 times) }\end{array}$ & $\begin{array}{l}\text { Whole graph in- } \\
\text { cluding DDS, FDS, } \\
\text { FFS }\end{array}$ & $\begin{array}{l}\text { Match predicted } \\
\text { links with the ac- } \\
\text { tual ones }\end{array}$ & $\begin{array}{l}\text { SP_t (the best } \\
\text { from evalua- } \\
\text { tion 1 over dis- } \\
\text { joint graph) } \\
\text { and RA (the } \\
\text { best from eval- } \\
\text { uation 1 over } \\
\text { joint graph) }\end{array}$ \\
\hline
\end{tabular}

384

385 Implementation

386 We have deployed the code and run all experiments on a server with RAM 64 GB, and Intel(R) Core(TM) i9-7980XE CPU @ 2.60GHz (18 Cores, 36 Threads). For DrugBank data preprocessing, we used Compute Canada cluster and to calculate SSP we used Google Colaboratory (a product from Google Research).

389 Our Proposed FDMine Framework

390

The FDMine framework (see Figure 2) is composed of several phases. In Phase 1, raw data is parsed from DrugBank and FooDB databases. In Phase 2, we execute two steps including a) building a homogenous network based on the structure similarity profile and b) updating the weights of the homogenous network using food compound contributions. Next, the graph is prepared with nodes representing drugs, food and food compounds' composition. In the graph, links are weighted by similarity and contribution scores (see Phase 3 in Figure 2). When applying the similarity thresholds, the homogenous network produces multiple subgraphs (disjoint graph). We build another version called the joint homogenous graph network and consider executing several link prediction algorithms including applied path category-based and neighborhoodbased similarity-based approaches. In the final Phase 4, we rank the newly predicted link (based on the score given by our methods), test the performance of the applied methods with the test dataset and finally, consult the literature to validate the top FDIs found using the different methods. For testing, we perform comparison using ground-truth and report literature validation for our leading findings (see Results and Discussion section). 


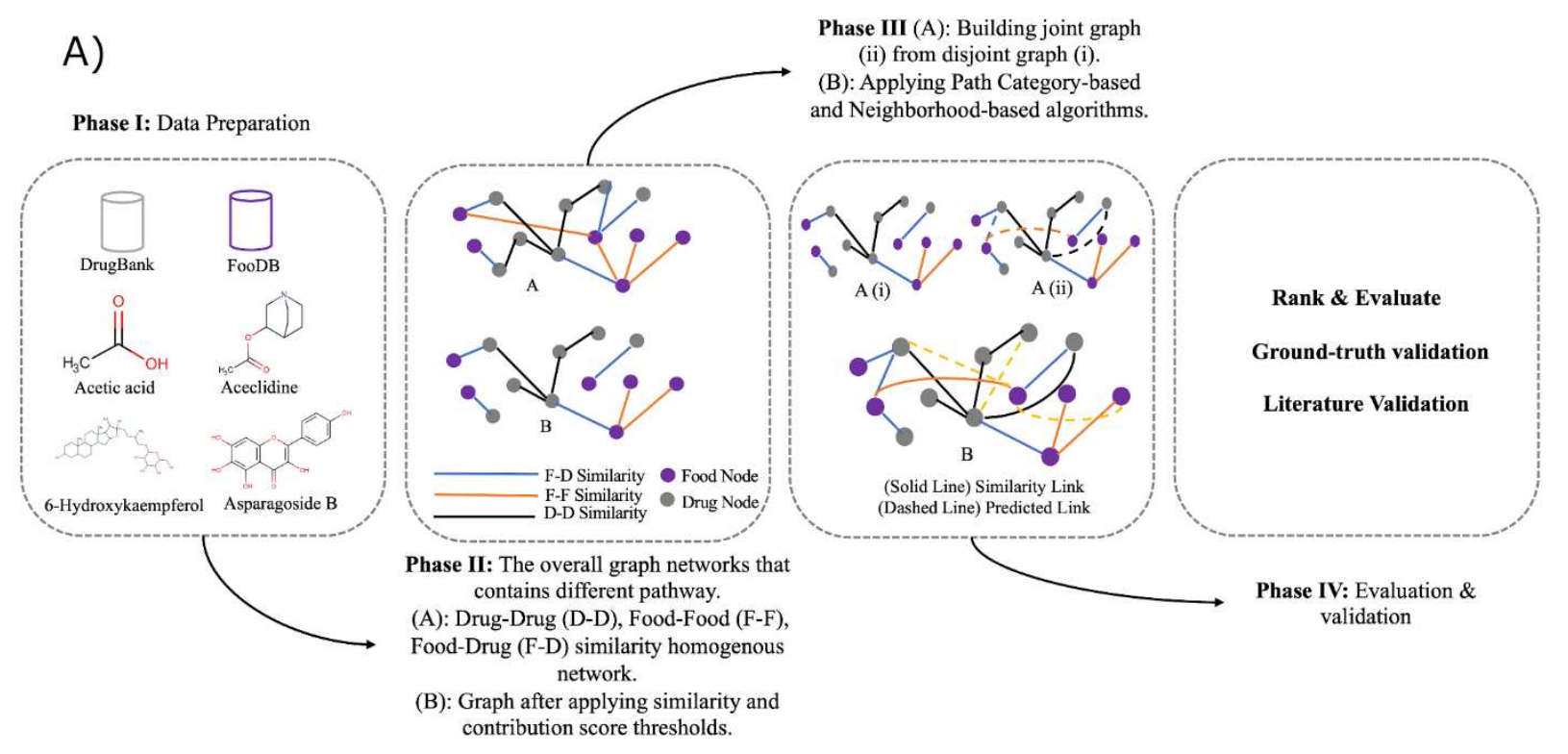

B)

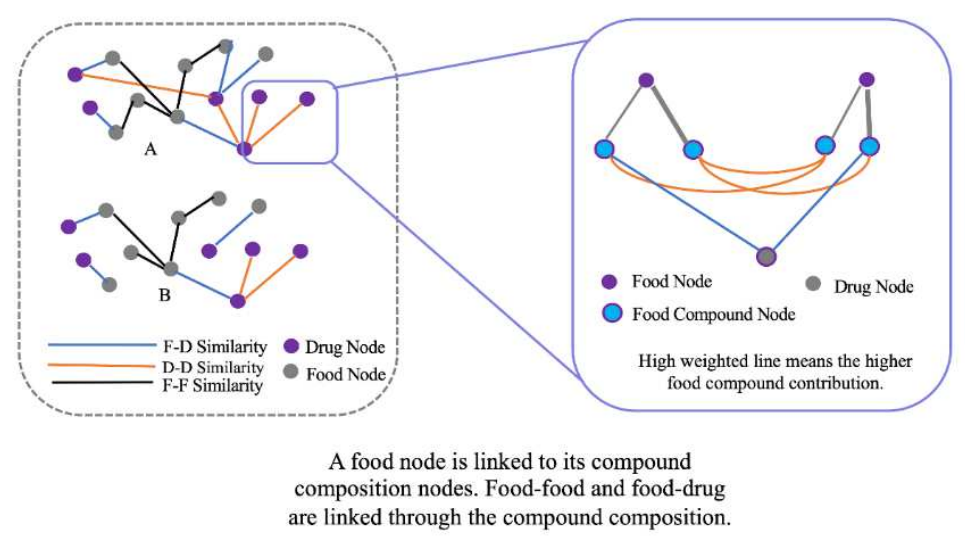

Figure 2 The framework of FDMine. The main steps are 1) preparing a comprehensive dataset describing FDIs by analyzing the whole DrugBank and FooDB databases with a unique representation of food composition 2) defining a scoring function for computing chemical compound contribution in food items, 3 ) implementing a set of path category-based (path length 2 and 3) and different neighborhood-based similarity-based algorithms to discover new FDIs from two different homogenous (disjoint and joint) graph networks, and 4) used the precision@k metric and calculated the precision@top (top 1\%, 2\%, and top 5\%) for drug-drug links to verify the accuracy of the algorithms with the test dataset.

\section{Results and Discussion}

413 The next subsections describe in detail the FDMine performance evaluation and the analysis of the novel 414 FDI predictions. 


\section{Prediction Results of FDMine}

416 Evaluation 1: Comprehensive evaluation for the recovery of DDS similarity links

417 As explained earlier, DDS similarity links are a priority in our evaluation setup as it establishes a ground 418 truth evaluation (see Evaluation 2 results). Here, drug-drug links are based on the similarity scorings we 419 computed. We have applied two different link prediction approaches over two different types of homoge420 nous graph networks. One is the disjoint graph network, and the other is the joint graph network. The 421 applied methods are the path category-based and neighborhood-based similarity-based link prediction al422 gorithms. We used path lengths 2 and 3 for the path category-based algorithm. SP_2 and SP_3 are used to 423 describe (Path length 2), and (Path length 3), respectively. From neighborhood-based similarity-based link 424 prediction, we applied Academic Adar (AA), Common Neighbor (CN), Jaccard Index (JAC), Dice Coeffi425 cient (Dice), Resource Allocation (RA), and Multiple paths of length l=3 (L3).

Table 4 provides a summary of different models over the disjoint graph network. For the disjoint graph, the SP_2 outperformed other methods. The precision rate for the top 1\% (i.e., precision@top-1) is 84\% for SP_2 while RA, the second best has achieved 64\%. For precision@top-2, SP_2 achieved the best results with $60 \%$ and L3, the second best $42 \%$. The highest value for the precision@ $@$ top-5 was achieved by the SP_2 (40\%). In the disjoint version of the graph, neighborhood-based similarity-based methods achieved, on average $17 \%$ with variant standard deviation each. However, SP_3 always showed a low performance (05\%,03\%,02\% for precision@top-1, precision@top-2, and precision@top-5 respectively) compared to all other methods. SP_2 achieved 52\% and 26\% AUC and PRC, respectively. All neighborhood-based similarity-based methods achieved more than $80 \%$ (AUC) except L3 which had a reported precision of $60 \%$. The PRC scores of the RA, AA, and $\mathrm{CN}$ were $70 \%, 65 \%$, and $60 \%$ respectively. When considering the joint version of the graph, different results were attained. The neighborhood-based similarity-based methods showed best results for the top precision@top-1, precision@top-2, and precision@top-5. For the precision@top-1, the RA achieved the best result (71\%), followed by AA (67\%). For the precision@top-2, L3 and RA both yielded similar performance (39\%). Additionally, all neighborhoodbased similarity-based methods produced the same result (16\%) for precision@ top-5. Contrary to the case of the disjoint version of the graph, the performance of SP_2 was weak. The SP_2 achieved, 23\%, 15\%, and 9\% for the precision@top-1, precision@top-2, and precision@top-5 respectively. For the joint graph, the neighborhood-based similarity-based algorithms achieved AUC of more than 90\% except L3 (65\%).

445 The value of the PRC is also high for the neighborhood-based similarity-based methods. The PRC scores 446 for the RA, AA, CN were 87\%, 86\%, and 84\% respectively. However, SP_3 always (disjoint and joint 
447 graphs) showed the weakest results in terms of all performance metrics (precision@ @ top, AUC, and PRC).

448 Table 5 summarizes the different models over the joint graph network. The comparison graph for the pre-

449 cision@top-1\%, precision@top-2\%, and precision@ top-5\% are provided in Figure 3. For more details, see

450 the Additional File 1 Figures S6 and S7.

451

452 Table 4 Comparison of the precision@top (average), AUC, PRC over eight different methods on the dis453 joint graph network

454

\begin{tabular}{|c|c|c|c|c|c|}
\hline Method & $\begin{array}{c}\text { 'Precision@Top- } \\
1(\%)\end{array}$ & $\begin{array}{c}\text { Precision@Top-2 } \\
(\%)\end{array}$ & $\begin{array}{c}\text { Precision@Top-5 } \\
(\%)\end{array}$ & $\begin{array}{c}\text { AUC } \\
(\%)\end{array}$ & $\begin{array}{c}\text { PRC } \\
(\%)\end{array}$ \\
\hline SP_2 & $84( \pm 6.3)$ & $60( \pm 5.3)$ & $40( \pm 2.5)$ & $\begin{array}{c}52 \\
( \pm 1.0)\end{array}$ & $26( \pm 1.0)$ \\
\hline SP_3 & $05( \pm 5.6)$ & $03( \pm 3.1)$ & $02( \pm 1.4)$ & $59( \pm 23.0)$ & $03( \pm 3.0)$ \\
\hline AA & $56( \pm 1.6)$ & $36( \pm 1.0)$ & $17( \pm 0.6)$ & $88( \pm .01)$ & $65( \pm 1.7)$ \\
\hline $\mathrm{CN}$ & $53( \pm 1.5)$ & $33( \pm 1.1)$ & $17( \pm 0.4)$ & $88( \pm 1.0)$ & $60( \pm 1.6)$ \\
\hline RA & $64( \pm 1.7)$ & $40( \pm 1.4)$ & $17( \pm 0.6)$ & $80( \pm 3.5)$ & $70( \pm 1.7)$ \\
\hline L3 & $58( \pm 1.9)$ & $42( \pm 1.2)$ & $17( \pm 0.6)$ & $60( \pm 4)$ & $30( \pm 3.1)$ \\
\hline JAC & $40( \pm 1.6)$ & $31( \pm 0.5)$ & $17( \pm 0.5)$ & $94( \pm 0.4)$ & $34( \pm 1.8)$ \\
\hline Dice & $40( \pm 1.6)$ & $31( \pm 0.5)$ & $17( \pm 0.5)$ & $97( \pm 0.7)$ & $35( \pm 2.0)$ \\
\hline
\end{tabular}

455

Table 5 Comparison of the precision@top (average), AUC, PRC over eight different methods on the joint

\begin{tabular}{|c|l|l|c|c|c|}
\hline Method & $\begin{array}{c}\text { Precision@Top-1 } \\
(\boldsymbol{\%})\end{array}$ & $\begin{array}{c}\text { Precision@Top-2 } \\
(\boldsymbol{\%})\end{array}$ & $\begin{array}{c}\text { Precision@Top-5 } \\
(\boldsymbol{\%})\end{array}$ & $\begin{array}{c}\text { AUC } \\
(\boldsymbol{\%})\end{array}$ & $\begin{array}{c}\text { PRC } \\
(\boldsymbol{\%})\end{array}$ \\
\hline SP 2 & $23( \pm 1.8)$ & $15( \pm 1.5)$ & $09( \pm 0.9)$ & $38( \pm 1)$ & $08( \pm 0.07)$ \\
\hline SP 3 & $0.1( \pm 0.2)$ & $0.1( \pm 0.1)$ & $0.1( \pm 0.0)$ & $88( \pm 31)$ & $00( \pm 0)$ \\
\hline
\end{tabular}




\begin{tabular}{|c|l|l|l|l|l|}
\hline $\mathrm{AA}$ & $67( \pm 0.9)$ & $37( \pm 0.5)$ & $16( \pm 0.2)$ & $95( \pm 0.6)$ & $86( \pm 0.6)$ \\
\hline $\mathrm{CN}$ & $65( \pm 0.9$ & $36( \pm 0.5)$ & $16( \pm 0.2)$ & $94( \pm 0.4)$ & $84( \pm 0.7)$ \\
\hline RA & $\mathbf{7 1 ( \pm \mathbf { 0 . 9 } )}$ & $\mathbf{3 9}( \pm \mathbf{0 . 5})$ & $\mathbf{1 6}( \pm \mathbf{0 . 2})$ & $92( \pm 2.4)$ & $\mathbf{8 7}( \pm \mathbf{1 . 9})$ \\
\hline L3 & $31( \pm 1.2)$ & $39( \pm 0.5)$ & $16( \pm 0.2)$ & $65( \pm 3.9)$ & $23( \pm 2.0)$ \\
\hline JAC & $59( \pm 0.7)$ & $35( \pm 0.4)$ & $16( \pm 0.2)$ & $97( \pm 0.3)$ & $66( \pm 1.4)$ \\
\hline Dice & $59( \pm 0.7)$ & $35( \pm 0.4)$ & $16( \pm 0.2)$ & $\mathbf{9 8}( \pm \mathbf{0 . 2})$ & $65( \pm 1.4)$ \\
\hline
\end{tabular}

459

460

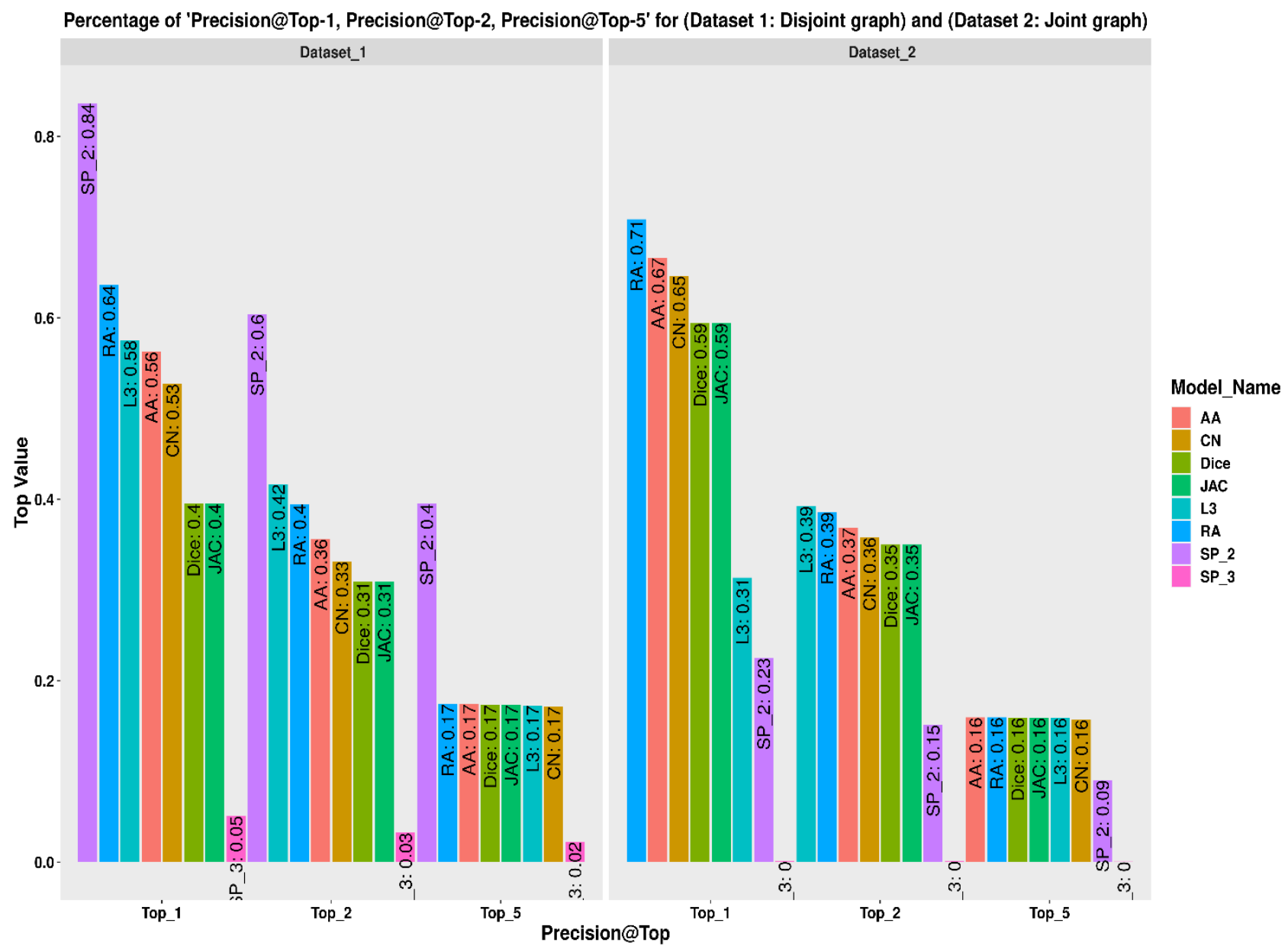

Figure 3 Comparison of the precision@ top over eight methods and two different graph networks 
464 The dataset we constructed using DrugBank and FooDB contains drug-drug links. The disjoint and joint dataset contains 2,926, and 6,581 drug-drug links respectively. From evaluation 1, out of 2,926, and 6,581, our method managed to discover 1,706, and 4,178 of those links respectively, reported as DDIs in the DrugBank. We have considered these 1,706 and 4,178 as known DDIs and as ground truth. To cross-validate the performance of FDMine we excluded a portion of known DDIs (or ground truth) as a test dataset from the main dataset and the rest of the dataset was used to train the models. Then, we calculate the precision@top-1\%, precision@top-2\%, and precision@top-5\% and found approximately the same performance of FDMine with the disjoint dataset and slightly better results for the joint dataset. Here, we have chosen only the best models, SP_2 for the disjoint dataset and RA for the joint dataset. Table 6 and Table 7 provides the performance of FDMine with the ground truth test dataset.

Table 6 Performance evaluation of ground truth using disjoint dataset and path category-based (path length-2) method

\begin{tabular}{|c|c|c|c|c|c|c|}
\hline Method & Proportion & \#Test DDI & \#Matched DDI & $\begin{array}{c}\text { Precision@ } \\
\text { Top-1 (\%) }\end{array}$ & $\begin{array}{c}\text { Precision@ } \\
\text { Top-2 (\%) }\end{array}$ & $\begin{array}{c}\text { Precision@ } \\
\text { Top-5 (\%) }\end{array}$ \\
\hline \multirow{3}{*}{ SP_2 } & 0.6 & 1023 & $864.8( \pm 13.85)$ & $84.49( \pm 5.09)$ & $72.29( \pm 6.59)$ & $47.11( \pm 4.00)$ \\
\cline { 2 - 7 } & 0.5 & 853 & $750.7( \pm 9.91)$ & $78.21( \pm 7.50)$ & $64.73( \pm 4.86)$ & $42.20( \pm 2.79)$ \\
\cline { 2 - 7 } & 0.4 & 682 & $613.5( \pm 6.06)$ & $76.31( \pm 5.77)$ & $57.51( \pm 5.53)$ & $36.81( \pm 3.88)$ \\
\cline { 2 - 7 } & 0.3 & 511 & $469.1( \pm 4.93)$ & $60.60( \pm 9.06)$ & $43.69( \pm 5.44)$ & $28.09( \pm 2.57)$ \\
\hline
\end{tabular}

Table 7 Performance evaluation of ground truth using joint dataset and Neighborhood-based Similaritybased (RA) Method

\begin{tabular}{|c|c|c|c|c|c|c|}
\hline Method & Proportion & \#Test DDI & \#Matched DDI & $\begin{array}{c}\text { Precision@ } \\
\text { Top-1 (\%) }\end{array}$ & $\begin{array}{c}\text { Precision@ } \\
\text { Top-2 (\%) }\end{array}$ & $\begin{array}{c}\text { Precision@ } \\
\text { Top-5 (\%) }\end{array}$ \\
\hline \multirow{3}{*}{ RA } & 0.6 & 2506 & $2413.0( \pm 9.12)$ & $94.93( \pm 0.30)$ & $93.16( \pm 0.71)$ & $51.55( \pm 0.71)$ \\
\cline { 2 - 7 } & 0.5 & 2089 & $2027.4( \pm 12.01)$ & $95.99( \pm 0.35)$ & $86.64( \pm 1.29)$ & $40.63( \pm 1.01)$ \\
\cline { 2 - 7 } & 0.4 & 1671 & $1628.4( \pm 6.97)$ & $96.75( \pm 0.49)$ & $72.15( \pm 1.07)$ & $31.64( \pm 0.54)$ \\
\cline { 2 - 7 } & 0.3 & 1253 & $1223.3( \pm 4.18)$ & $90.96( \pm 1.05)$ & $54.59( \pm 0.86)$ & $22.97( \pm 0.43)$ \\
\hline
\end{tabular}

482 Here we randomly assigned $30 \%$ of all (DD, FF, FD) links from the whole dataset to make the test dataset, 483 and the rest of the $70 \%$ was used to train the model. We applied 'shortest path length 2' over the disjoint 484 and 'RA' over joint graph. The 30\% test dataset from the disjoint and joint dataset contains 26,157 and 48527,612 links respectively. The FDMine was able to recover an average of $9612.6( \pm 5723.06)$ and 27448.4 
$( \pm 14.20)$ links respectively from the disjoint and joint dataset using 'shortest path length 2 ' and 'RA' meth487 ods respectively.

\section{New Food Drug Interaction Prediction}

After comparing the different approaches for link prediction, we executed the FDMine framework to find top candidates for FDIs. In the framework, we consider taking the top results from the joint and disjoint versions. At the final stage of FDMine, we surveyed the literature to find supporting evidence to the generated predictions. We have performed two batches using different contribution scores (i.e., 0.5 and 0.3 , respectively). The default value in the FDMine framework is a 0.5 contribution score. The results as listed in Additional file 2, have shown some repeated drugs in top findings due to a higher threshold value. A high threshold value will lead to removing more connections in the graph. This will lead to more disjoint subgraphs and nodes with higher connections within the subgraphs gain higher rank scores. Therefore, we consider a more relaxed threshold and generate Batch-2 results (i.e., contribution score of 0.3). In this batch, we see more diversity in results. Additional file 2 lists all Batch-1 results, and Additional file 3 lists all Batch-2 results with a description of the experiments used in each. We analyzed all results of both batches and discussed here the insights driven from two types of evidence including: 1) linking food to anti-inflammatory effects based on known biological pathways and 2) linking food to pharmacological effects based on matching functions of a drug and a chemical substructure found in food.

504

Food compound compositions with Anti-inflammatory effects (biological pathway driven evidence)

506

The results in this section are part of Batch-1 results (see Additional file 2). Our findings using a literature review indicate possible pairing of drug and nutraceutical food components. As per the summary in Table thesis for inflammatory response [72], beta-adrenergic signaling for cardiac output modulation [73] and

511 GABA pathway [74] - a GABA based inhibitory neurotransmitter that down-regulates CNS stimulation

512 [75]. After examining the results in Table 8, we have found that dietary fatty acids like Oleic acid 513 (FDB012858), Erucic acid (FDB004287), (Z,Z)-9,12-Octadecadienoic (FDB012760) and Elaidic acid 514 (FDB002951) available in foods like Onions - FOOD00006, Garden Cress - FOOD00099, Pomegranate515 FOOD00151, etc. can affect prostaglandin biosynthesis via PPAR mediated mechanism and Gabaergic 516 pathway. Figures 4 and 5 highlight the list of these compounds and their interaction with Peroxisome pro- 
517 liferator-activated receptor (PPAR) and GABA-mediated effects, respectively. Similarly, we found evi518 dence of food components like Eugenol (FDB012171), Carvacrol (FDB014512), which can potentially 519 substantiate hypotensive effects when taken with beta adrenergic drugs. For example, Eugenol has been 520 known to cause vasodilation via vanilloid TRPV4 receptors found on endothelial muscles in arteries. Beta521 adrenergic drugs are prescribed to patients suffering from hypertension to decrease blood pressure (BP). 522 So, when combined, this can cause an elevated drop in BP.

523

524 Prostaglandins are compounds that play a role in the anti-inflammatory pathway during injury [76]. An 525 essential molecular building block in humans is arachidonic acid. It interacts with the Peroxisome prolifer526 ator-activated receptor (PPAR) to form various prostaglandins [76] or anti-inflammatory compounds. Var527 ious dietary fatty acids (see Table 8; Oleic acid, Linoleic acid, Erucic Acid, Eldaic acid) are also absorbed 528 via the exogenous chylomicron pathway and hydrolysed for various tissues to absorb them for further processing [77]. Some of our predicted compound items include Oleic acid - FDB012858, and Erucic acid FDB004287, that are similar to Arachidonic acid and are analogous [78] structures, belonging to the fatty acid group and are found in many dietary sources including Celery - FOOD00015, Peanuts (FOOD00016) and Burdock - FOOD00017 (See Table 8). Our literature review has highlighted reported evidence on the influence of these dietary fatty acids on the Arachidonic acid cycle. Arachidonic acid is a precursor for the 534 synthesis of various other biomolecules, associated with anti-inflammatory pathways [79]. During injury, inflammation occurs and causes arachidonic acid to bind with PPAR-gamma receptors as shown in Figure 4 to form prostaglandins or protective anti-inflammatory agents to curb the injury [80]. Fatty acids (see Table 8) also compete with arachidonic acid during injury or inflammation to produce various substituted prostaglandins belonging to a family of derivative compounds known as eicosanoids [81], via PPAR [82].

539 Since the substituted prostaglandins are not exactly derived from arachidonic acid, they show slightly fewer 540 anti-inflammatory profiles as compared to other eicosanoids produced directly from arachidonic acid [83].

541 It is worth noting that arachidonic acid belongs to the list of essential fatty acids including alpha-linoleic

542 acid and docosahexaenoic acid [83]. There has been evidence to show that dietary sources such Linoleic 543 acid, Erucic acid and Elaidic acid (see Table 8) did increase PPAR gene expression in healthy subjects [84]. 544 In 2012 Hung-Tsung Wu et al. also showed the interaction of oleic acid with PPAR-g receptors [85]. These 545 results may suggest taking drugs like Doconexent - DB03756 with foods such as FOOD00099 - Garden 546 Cress, FOOD00151 - Pomegranate, FOOD00009 - Chives, FOOD00062 - Hazelnut, FOOD00525 - Maca547 damia can alter the normal dynamics of anti-inflammatory responses. Arachidonic acid is biosynthesized 548 from dietary linoleic acid and released by phospholipases during inflammation. This pathway is also known 549 as the COX or Cyclooxygenase pathway [86]. 
551 Table 8 Depicts some of our top correlations of food substances that can potentially be involved in food

552 drug interactions when combined with a drug with a similar activity. Each food component can link to any of the drugs as long as they are in the same batch.

\begin{tabular}{|c|c|c|c|c|c|c|}
\hline $\begin{array}{l}\text { Food com- } \\
\text { ponent }\end{array}$ & $\begin{array}{c}\text { Food source } \\
\text { ID }\end{array}$ & $\begin{array}{l}\text { Food } \\
\text { name }\end{array}$ & $\begin{array}{c}\text { Pharmacological ac- } \\
\text { tions }\end{array}$ & Drug & $\begin{array}{l}\text { Refer- } \\
\text { ences }\end{array}$ & Batch \\
\hline \multirow[t]{10}{*}{ Oleic acid } & FOOD00006 & $\begin{array}{l}\text { Garden } \\
\text { Onion }\end{array}$ & \multirow{13}{*}{$\begin{array}{l}\text { Dietary fatty acids like } \\
\text { Oleic acid can compete } \\
\text { with arachidonic acid } \\
\text { by interacting with } \\
\text { PPAR-g receptor to } \\
\text { form prostaglandins }\end{array}$} & \multirow{13}{*}{$\begin{array}{l}\text { Vigabatrin, } \\
\text { Pregabalin, } \\
\text { Gabapentin } \\
\text { Doconexent }\end{array}$} & \multirow{13}{*}{$\begin{array}{l}{[82,} \\
85,89, \\
90,91, \\
92]\end{array}$} & \multirow{13}{*}{$\begin{array}{l}\text { Top } 10 \text { in } \\
\text { joint and } \\
\text { disjoint - } \\
\text { batch } 1 \\
\text { (supple- } \\
\text { mentary } \\
\text { file 2) }\end{array}$} \\
\hline & FOOD00009 & Chives & & & & \\
\hline & FOOD00011 & $\begin{array}{l}\text { Cashew } \\
\text { Nus }\end{array}$ & & & & \\
\hline & FOOD00012 & $\begin{array}{l}\text { Pineap- } \\
\text { ple }\end{array}$ & & & & \\
\hline & FOOD00015 & $\begin{array}{l}\text { Wild } \\
\text { celery }\end{array}$ & & & & \\
\hline & FOOD00016 & Peanuts & & & & \\
\hline & FOOD00017 & $\begin{array}{l}\text { Bur- } \\
\text { dock }\end{array}$ & & & & \\
\hline & FOOD00021 & $\begin{array}{l}\text { Aspara- } \\
\text { gus }\end{array}$ & & & & \\
\hline & FOOD00024 & $\begin{array}{l}\text { Brazil } \\
\text { Nut }\end{array}$ & & & & \\
\hline & FOOD00026 & Borage & & & & \\
\hline Erucic acid & FOOD00099 & $\begin{array}{l}\text { Garden } \\
\text { Cress }\end{array}$ & & & & \\
\hline Elaidic acid & FOOD00151 & $\begin{array}{l}\text { Pome- } \\
\text { granate }\end{array}$ & & & & \\
\hline $\begin{array}{l}(\mathrm{Z}, \mathrm{Z})-9,12- \\
\text { Octadecadi- } \\
\text { enoic acid }\end{array}$ & FOOD00009 & & & & & \\
\hline Eugenol & FOOD00179 & Cloves & $\begin{array}{l}\text { Euginol causes vasodi- } \\
\text { lation via vanilloid }\end{array}$ & $\begin{array}{l}\text { Betaxolol, } \\
\text { Atenelol, }\end{array}$ & $\begin{array}{l}{[86,} \\
87]\end{array}$ & $\begin{array}{l}\text { Top } 20 \text { in } \\
\text { joint and }\end{array}$ \\
\hline
\end{tabular}




\begin{tabular}{|c|c|c|c|c|c|}
\hline & & & $\begin{array}{l}\text { TRPV4 receptors } \\
\text { found on endothelial } \\
\text { muscles found on ar- } \\
\text { teries. Euginol \& Cap- } \\
\text { sacin have a vanilloid } \\
\text { ring. TRPV4 is in- } \\
\text { volved in BP regula- } \\
\text { tion via various mech- } \\
\text { anisms }\end{array}$ & $\begin{array}{l}\text { Esmolol, } \\
\text { Bisprolol, } \\
\text { Metoprolol }\end{array}$ & $\begin{array}{l}\text { disjoint - } \\
\text { batch } 2 \\
\text { (supple- } \\
\text { mentary } \\
\text { file 3) }\end{array}$ \\
\hline $\begin{array}{c}\text { Isopropyl-2- } \\
\text { methylphe- } \\
\text { nol }\end{array}$ & FOOD00089 & Hyssop & $\begin{array}{l}\text { p-Cymene has been } \\
\text { reported to cause } \\
\text { smooth muscle vasodi- } \\
\text { lation and has antihy- } \\
\text { pertensive effects }\end{array}$ & & \\
\hline $\begin{array}{c}\text { 1-Isopropyl- } \\
4- \\
\text { methylben- } \\
\text { zene }\end{array}$ & FOOD00013 & Dill & $\begin{array}{l}\text { Also known as p-cy- } \\
\text { mene. It has been } \\
\text { shown to cause seda- } \\
\text { tive effects via GABA } \\
\text { adrenergic receptors } \\
\text { and also causes vaso- } \\
\text { dilation of smooth ar- } \\
\text { terial muscles }\end{array}$ & & \\
\hline \multirow{2}{*}{$\begin{array}{l}\text { 1-Methoxy- } \\
\text { 4-(2-pro- } \\
\text { penyl)ben- } \\
\text { zene }\end{array}$} & FOOD00137 & Anise & \multirow{2}{*}{$\begin{array}{l}\text { Methyl Chavicol has } \\
\text { been reported as an } \\
\text { adjunct therapy for } \\
\text { treatment of hyperten- } \\
\text { sion, found in anise. }\end{array}$} & & \\
\hline & FOOD00019 & $\begin{array}{c}\text { Tarra- } \\
\text { gon }\end{array}$ & & & \\
\hline
\end{tabular}




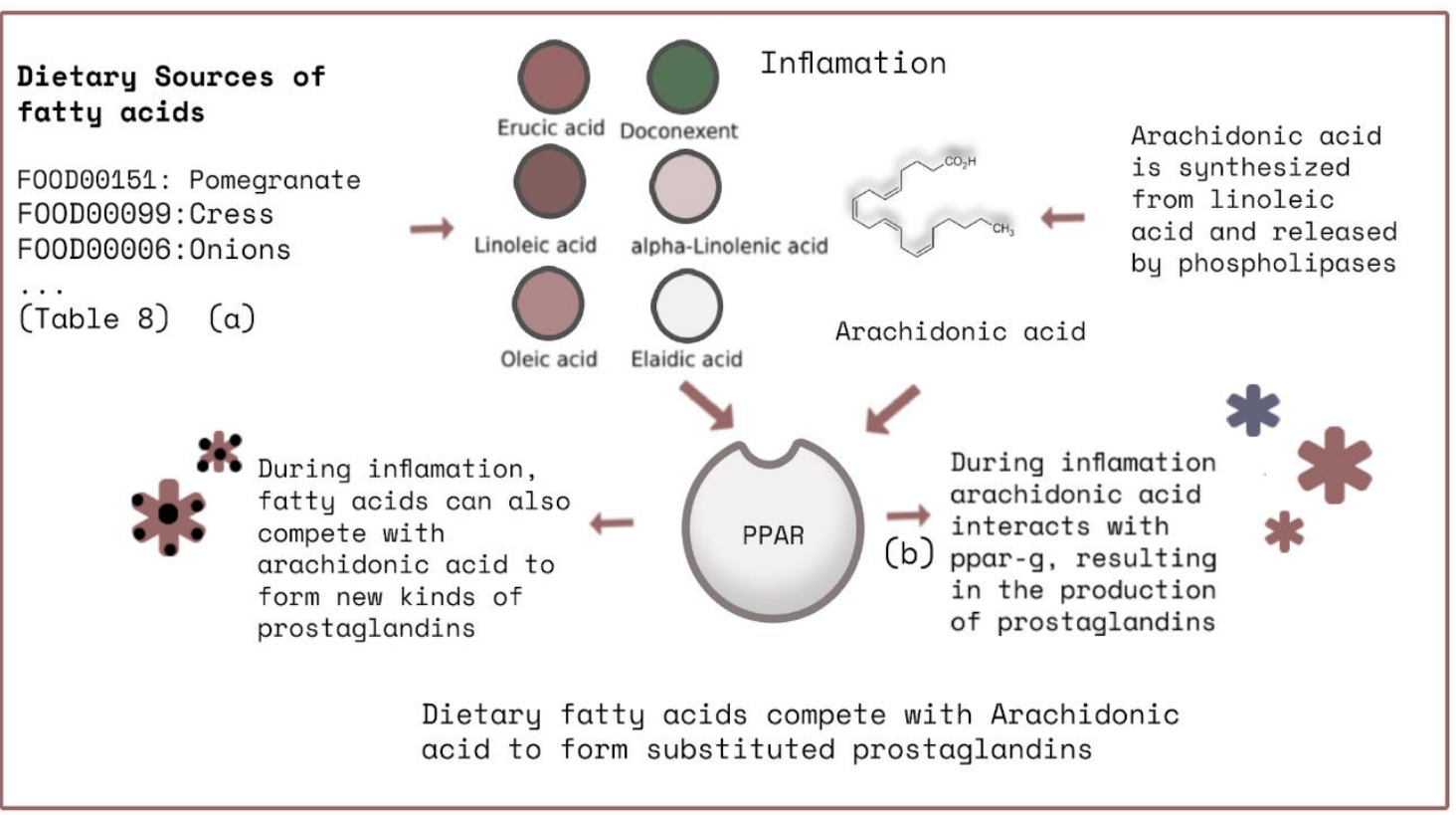

Figure 4 An illustration depicting the effect of dietary fatty acids on COX pathway a) Various foods are rich sources of dietary fatty acids b) During inflammation, Arachidonic acid interacts with PPAR to produce prostaglandins c) Dietary Fatty acids can compete with Arachidonic acid during inflammation at PPAR to form substituted prostaglandin variants.

\section{Food compound composition with pharmacological effects (similar function-driven evidence)}

Here, we relaxed the contribution score to 0.3 (i.e., Batch-2) to obtain a diverse set of results (see Additional file 3). In this part of our literature validation, we analyze the potential of similar functions of drugs and food compounds on specific diseases. The results in Table 8 highlight some correlations with a group of drugs called beta-adrenergic drugs and essential oils. Our top correlated pairs of food and drug observed that both of them caused reduced blood pressure. Beta-blockers are used to treat hypertension in patients. Beta-blockers consist of b1, b2, and b3 subtype receptors. Beta-blockers can fall into various categories based on the extent of selectivity of binding across these subtypes. For example, Atenolol (DB00335), Bisoprolol (DB00612), Metoprolol (DB00264) and Esmolol (DB00187) are b1 selective blockers [88]. The effects of b1 blockade include a decrease in cardiac output by inhibiting the SA and AV nodes, thereby decreasing stroke volume [89]. Propranolol (DB00571) and Penbutolol (DB01359), on the other hand, are non-selective beta-adrenergic blockers. Studies have also observed that beta-blockers may also contribute to GABA turnover in the cerebrum [90]. 
577 The results suggest that beta-blocker drugs like Atenolol, Betaxolol, Esmolol, Oxprenolol, Penbutalol, and

578 Propranolol can interact in the form of synergism when combined with a specific compound composition 579 including p-Cymene - FDB014512, Eugenol - FDB012171, and Carvacrol (terpenoid substances). For ex-

580 ample, Marcio et al. 2011 reported that monoterpenoids like p-Cymene - FDB014512 and Carvacrol have 581 vasorelaxant effects [86].

582

583 We were able to confirm that fatty acids (Oleic acid (FDB012858), Erucic acid (FDB004287), (Z,Z)-9,12-

584 Octadecadienoic (FDB012760) and Elaidic acid (FDB002951) ) can cross the blood-brain barrier and be 585 beneficial to relieve anxiety [91]. They are also believed to act via stimulation of GABA-A based receptors.

586 Benzodiazepines, barbiturates [92] and some anticonvulsants act by modulating the GABA receptors [93].

587 The inhibitory effects of GABA help relieve seizures. However, drugs like Pregabalin and Gabapentin in-

588 stead act by blocking calcium or sodium channels to help stabilize seizures. Although this is not directly 589 interacting with GABA receptors, it helps reduce excitatory neurotransmitters. Thus, they may help sub590 stantiate antiepileptic activity by increasing amounts of GABA.

591

592

593

594 Figure 5 An illustration depicting Gabaergic drug mechanisms. Dietary sources containing fatty acids in595

596 crease the production of GABA. Taking drugs like Vigabatrin, pregabalin \& Gabapentin with such a diet can increase Gabaergic effects. 
598 In summary, the discussed pairs of food ingredients and drugs can influence their own pharmacokinetics.

599 For example, taking beta-adrenergic drugs with food containing terpenes like Eugenol and Methyl chavicol

600 can potentially cause more pronounced antihypertensive effects. Taking antiepileptic medications along

601 with foods containing fatty acids can potentially elevate overall GABA levels significantly than when they

602 are taken individually. Moreover, dietary fatty acids can also interact with the PPAR receptor during in-

603 flammation to produce variations of prostaglandins. This demonstrates the feasibility of using our FDMine

604 framework to identify potential food and drug interactions.

\section{Conclusion}

606 In this study, we introduced FDMine as a framework to infer the interaction between food compounds and

607 drugs using a homogenous graph representation. We considered several resources to construct food-drug,

608 drug-drug, and food-food similarity profiles. FDMine uses established path category-based and neighbor-

609 hood-based similarity methods to predict FDIs efficiently. A subset of Drug-drug interactions was used as

610 ground-truth evaluations. This proposed methodology is based on encoding all entities including drug and

611 food into a homogenous graph of chemical nodes. Therefore, any part of this graph can then be used as a

612 representative evaluation, potentially informative to clinicians and researchers. We have performed addi-

613 tionally two types of evaluations to benchmark results using different parts of the graph. The shortest path-

614 based method has achieved a precision $84 \%, 60 \%$ and $40 \%$ for the top $1 \%, 2 \%$ and $5 \%$, respectively.

615 FDMine was able to achieve an average 99.4\% recovery rate from 27,612 available links in the joint version

616 of the graph. We validated the top FDIs predicted using FDMine to demonstrate the applicability of the

617 model. In the literature validation, we discussed the therapeutic effects of a group of food items. We ob-

618 served that a set of FDIs may reduce blood pressure, have anti-inflammatory effects or reduce seizure. The

619 benchmark results and literature review suggest that FDMine can help to identify FDIs precisely and may

620 represent an advanced strategy in drug discovery.

621

622 Availability of data and materials

623 The code and datasets supporting the conclusions of this article are included within the article (and its

624 additional files) or is made available at https://github.com/mostafiz67/FDMine_Framework

\section{Competing interests}

626 The authors declare that they have no competing interests. 


\section{Author contributions}

628 MR and OS conceptualized the problem. MR was responsible for solution development and implementa629 tion. SV and AM were responsible for validating the new predictions. AM, JL and OS reviewed the text 630 and the evaluation of the work. JL and OS supervised the study.

631

\section{Funding information}

633 This work was supported by a Natural Science and Engineering Research Council of Canada, Canada Re634 search Chair grant (grant number 231266) to JL, a Canada Foundation for Innovation and Nova Scotia 635 Research and Innovation Trust infrastructure grant to JL, and a Natural Science and Engineering Research 636 Council of Canada Discovery Grant to JL. This research was supported in part by the Heaps Chair Endow637 ment Fund at St. Francis Xavier University through the Dr. H. Stanley \& Doreen Alley Heaps Chairship. 638 This research was enabled in part by support provided by Compute Canada (www.computecanada.ca) and 639 by Google Cloud under the GCP research credits program.

\section{Acknowledgements}

642 Authors would like to acknowledge Sumaiya Amin for some preliminary data preparation in this work.

643

\section{References}

645 1. Bushra, R., Aslam, N., Khan, A.Y.: Food-drug interactions. Oman medical journal 26(2), 77 (2011)

646 2. Schmidt, L.E., Dalhoff, K.: Food-drug interactions. Drugs 62(10), 1481-1502 (2002)

647 3. Won, C.S., Oberlies, N.H., Paine, M.F.: Mechanisms underlying food-drug interactions: inhibition of 648

649

650

651

652

653

654

655 intestinal metabolism and transport. Pharmacology \& therapeutics 136(2), 186-201 (2012)

4. Mouly, S., Morgand, M., Lopes, A., Lloret-Linares, C., Bergmann, J.: Drug-food interactions in internal medicine: What physicians should know? La Revue de medecine interne 36(8), 530-539 (2015)

5. Ased, S., Wells, J., Morrow, L.E., Malesker, M.A.: Clinically significant food-drug interactions. The Consultant Pharmacist ${ }^{\circledR}$ 33(11), 649-657 (2018)

6. Hollander, A.A., van Rooij, J., Lentjes, E.G., Arbouw, F., van Bree, J.B., Schoemaker, R.C., van Es, L.A., van der Woude, F.J., Cohen, A.F.: The effect of grapefruit juice on cyclosporine and prednisone metabolism in transplant patients. Clinical Pharmacology \& Therapeutics 57(3), 318-324 (1995) 
7. Dahan, A., Altman, H.: Food-drug interaction: grapefruit juice augments drug bioavailability-mechanism, extent and relevance. European journal of clinical nutrition 58(1), 1-9 (2004)

8. Koziolek, M., Alcaro, S., Augustijns, P., Basit, A.W., Grimm, M., Hens, B., Hoad, C.L., Jedamzik, P., Madla, C.M., Maliepaard, M., et al.: The mechanisms of pharmacokinetic food-drug interactions-a perspective from the ungap group. European Journal of Pharmaceutical Sciences 134, 31-59 (2019)

9. Goldstein, L.H., Elias, M., Ron-Avraham, G., Biniaurishvili, B.Z., Madjar, M., Kamargash, I., Braunstein, R., Berkovitch, M., Golik, A.: Consumption of herbal remedies and dietary supplements amongst patients hospitalized in medical wards. British journal of clinical pharmacology 64(3), 373-380 (2007)

665

10. Berkovich, L., Earon, G., Ron, I., Rimmon, A., Vexler, A., Lev-Ari, S.: Moringa oleifera aqueous leaf extract down-regulates nuclear factor-kappab and increases cytotoxic effect of chemotherapy in pancreatic cancer cells. BMC complementary and alternative medicine 13(1), 1-7 (2013)

11. Hermawan, A., Nur, K.A., Dewi, D., Putri, P., Meiyanto, E., et al.: Ethanolic extract of moringa oleifera

669 increased cytotoxic effect of doxorubicin on hela cancer cells. Journal of Natural remedies 12(2), 108-114 (2012)

670

671

12. Al-Asmari, A.K., Albalawi, S.M., Athar, M.T., Khan, A.Q., Al-Shahrani, H., Islam, M.: Moringa oleifera as an anti-cancer agent against breast and colorectal cancer cell lines. PloS one 10(8), 0135814 (2015)

672

673

13. Nirmala, M.J., Samundeeswari, A., Sankar, P.D., et al.: Natural plant resources in anti-cancer therapy-a review. Res Plant Biol 1(3), 01-14 (2011)

14. Mouly, S., Lloret-Linares, C., Sellier, P.-O., Sene, D., Bergmann, J.-F.: Is the clinical relevance of drug-food and drug-herb interactions limited to grapefruit juice and saint-john's wort? Pharmacological research 118, 82-92 (2017)

15. de Boer, A., Van Hunsel, F., Bast, A.: Adverse food-drug interactions. Regulatory Toxicology and Pharmacology 73(3), 859-865 (2015)

16. Segal, E.M., Flood, M.R., Mancini, R.S., Whiteman, R.T., Friedt, G.A., Kramer, A.R., Hofstetter, M.A.: Oral chemotherapy food and drug interactions: a comprehensive review of the literature. Journal of oncology practice 10(4), 255-268 (2014)

683

17. Di Minno, A., Frigerio, B., Spadarella, G., Ravani, A., Sansaro, D., Amato, M., Kitzmiller, J.P., Pepi, M., Tremoli, E., Baldassarre, D.: Old and new oral anticoagulants: food, herbal medicines and drug interactions. Blood reviews 31(4), 193-203 (2017) antidiabetic drugs and herbs: an overview of mechanisms of action and clinical implications. Diabetology \& metabolic syndrome 9(1), 1-12 (2017)

19. Stephenson, N., Shane, E., Chase, J., Rowland, J., Ries, D., Justice, N., Zhang, J., Chan, L., Cao, R.: Survey of machine learning techniques in drug discovery. Current drug metabolism 20(3), 185-193 (2019)

20. Lee, G., Park, C., Ahn, J.: Novel deep learning model for more accurate prediction of drug-drug interaction effects. BMC bioinformatics 20(1), 1-8 (2019) 
21. Ryu, J.Y., Kim, H.U., Lee, S.Y.: Deep learning improves prediction of drug-drug and drug-food interactions. Proceedings of the National Academy of Sciences 115(18), 4304-4311 (2018)

22. Reker, D., Shi, Y., Kirtane, A.R., Hess, K., Zhong, G.J., Crane, E., Lin, C.-H., Langer, R., Traverso, G.: Machine learning uncovers food-and excipient-drug interactions. Cell reports 30(11), 3710-3716 (2020)

23. Allahgholi, M., Rahmani, H., Javdani, D., Weiss, G., M’odos, D.: Addi: Recommending alternatives for drug-drug interactions with negative health effects. Computers in Biology and Medicine 125, 103969 (2020)

24. Feng, Y.-H., Zhang, S.-W., Shi, J.-Y.: Dpddi: a deep predictor for drug-drug interactions. BMC bioinformatics 21(1), 1-15 (2020)

25. You, J., McLeod, R.D., Hu, P.: Predicting drug-target interaction network using deep learning model. Computational biology and chemistry 80, 90-101 (2019)

26. Ba-Alawi, W., Soufan, O., Essack, M., Kalnis, P., Bajic, V.B.: Daspfind: new efficient method to predict drug-target interactions. Journal of cheminformatics 8(1), 1-9 (2016)

704

27. Olayan, R.S., Ashoor, H., Bajic, V.B.: Ddr: efficient computational method to predict drug-target interactions using graph mining and machine learning approaches. Bioinformatics 34(7), 1164-1173 (2018)

706

707

708

709

710

711

712

713

714

715

716

717

718

719

720

721

722

723

724

725

726

28. Lu, Y., Guo, Y., Korhonen, A.: Link prediction in drug-target interactions network using similarity indices. BMC bioinformatics 18(1), 1-9 (2017)

29. Fokoue, A., Sadoghi, M., Hassanzadeh, O., Zhang, P.: Predicting drug-drug interactions through large-scale similarity-based link prediction. In: European Semantic Web Conference, pp. 774-789 (2016). Springer

30. Naveja, J.J., Rico-Hidalgo, M.P., Medina-Franco, J.L.: Analysis of a large food chemical database: Chemical space, diversity, and complexity. F1000Research 7 (2018)

31. FooDB: Foodb version 1.0 (2017)

32. Wishart, D.S., Knox, C., Guo, A.C., Shrivastava, S., Hassanali, M., Stothard, P., Chang, Z., Woolsey, J.: Drugbank: a comprehensive resource for in silico drug discovery and exploration. Nucleic acids research 34(suppl 1), 668-672 (2006)

33. Wishart, D.S., Feunang, Y.D., Guo, A.C., Lo, E.J., Marcu, A., Grant, J.R., Sajed, T., Johnson, D., Li, C., Sayeeda, Z., et al.: Drugbank 5.0: a major update to the drugbank database for 2018. Nucleic acids research 46(D1), 1074-1082 (2018)

34. Wishart, D.S., Knox, C., Guo, A.C., Cheng, D., Shrivastava, S., Tzur, D., Gautam, B., Hassanali, M.: Drugbank: a knowledgebase for drugs, drug actions and drug targets. Nucleic acids research 36(suppl 1), 901-906 (2008)

35. Bajusz, D., R'acz, A., H’eberger, K.: Why is tanimoto index an appropriate choice for fingerprint-based similarity calculations? Journal of cheminformatics 7(1), 1-13 (2015)

36. Alazmi, M., Kuwahara, H., Soufan, O., Ding, L., Gao, X.: Systematic selection of chemical fingerprint features improves the gibbs energy prediction of biochemical reactions. Bioinformatics 35(15), 2634-2643 (2019) 
37. Morgan, H.L.: The generation of a unique machine description for chemical structures-a technique developed at chemical abstracts service. Journal of Chemical Documentation 5(2), 107-113 (1965)

38. Rogers, D., Hahn, M.: Extended-connectivity fingerprints. Journal of chemical information and modeling $50(5), 742-754(2010)$

39. Awale, M., Reymond, J.-L.: Web-based tools for polypharmacology prediction. In: Systems Chemical Biology, pp. 255-272. Springer, (2019)

40. Awale, M., Reymond, J.-L.: Polypharmacology browser ppb2: target prediction combining nearest neighbors with machine learning. Journal of chemical information and modeling 59(1), 10-17 (2018)

735

41. Riniker, S., Landrum, G.A.: Open-source platform to benchmark fingerprints for ligand-based virtual screening. Journal of cheminformatics 5(1), 1-17 (2013)

42. Rai, A., Kumar, V., Jerath, G., Kartha, C., Ramakrishnan, V.: Mapping drug-target interactions and synergy in multi-molecular therapeutics for pressure-overload cardiac hypertrophy. NPJ systems biology and applications 7(1), 1-11 (2021)

43. Gottlieb, A., Stein, G.Y., Oron, Y., Ruppin, E., Sharan, R.: Indi: a computational framework for inferring drug interactions and their associated recommendations. Molecular systems biology 8(1), 592 (2012)

44. Vilar, S., Harpaz, R., Uriarte, E., Santana, L., Rabadan, R., Friedman, C.: Drug-drug interaction through molecular structure similarity analysis. Journal of the American Medical Informatics Association 19(6), 1066-1074 (2012)

45. Kov’acs, I.A., Luck, K., Spirohn, K., Wang, Y., Pollis, C., Schlabach, S., Bian, W., Kim, D.-K., Kishore, N., Hao, T., et al.: Network-based prediction of protein interactions. Nature communications 10(1), 1-8 (2019)

46. Al Hasan, M., Chaoji, V., Salem, S., Zaki, M.: Link prediction using supervised learning. In: SDM06: Workshop on Link Analysis, Counter-terrorism and Security, vol. 30, pp. 798-805 (2006)

47. Guimer`a, R., Sales-Pardo, M.: Missing and spurious interactions and the reconstruction of complex network. Proceedings of the National Academy of Sciences 106(52), 22073-22078 (2009)

751

752

753

754

755

756

757

758

759

760

761
48. Chen, H., Li, X., Huang, Z.: Link prediction approach to collaborative filtering. In: Proceedings of the 5th ACM/IEEE-CS Joint Conference on Digital Libraries (JCDL'05), pp. 141-142 (2005). IEEE

49. Clauset, A., Moore, C., Newman, M.E.: Hierarchical structure and the prediction of missing links in networks. Nature 453(7191), 98-101 (2008)

50. Folino, F., Pizzuti, C.: Link prediction approaches for disease networks. In: International Conference on Information Technology in Bio-and Medical Informatics, pp. 99-108 (2012). Springer

51. Daminelli, S., Thomas, J.M., Dur'an, C., Cannistraci, C.V.: Common neighbours and the local-communityparadigm for topological link prediction in bipartite networks. New Journal of Physics 17(11), 113037 (2015)

52. Adamic, L.A., Adar, E.: Friends and neighbors on the web. Social networks 25(3), 211-230 (2003)

53. Liben-Nowell, D., Kleinberg, J.: The link-prediction problem for social networks. Journal of the American society for information science and technology 58(7), 1019-1031 (2007)

31 of 35 
54. Jaccard, P.: 'E comparative study of floral distribution in a portion of the alps and jura. Bull Soc Vaudoise Sci Nat 37, 547-579 (1901)

55. Zhou, T., Lü, L., Zhang, Y.-C.: Predicting missing links via local information. The European Physical Journal B71(4), 623-630 (2009)

56. Yang, Y., Lichtenwalter, R.N., Chawla, N.V.: Evaluating link prediction methods. Knowledge and Information Systems 45(3), 751-782 (2015)

57. Chen, Y., Wang, W., Liu, J., Feng, J., Gong, X.: Protein interface complementarity and gene duplication improve link prediction of protein-protein interaction network. Frontiers in genetics 11 (2020)

58. Dice, L.R.: Measures of the amount of ecologic association between species. Ecology 26(3), 297-302 (1945)

59. Sorensen, T.A.: A method of establishing groups of equal amplitude in plant sociology based on similarity of species content and its application to analyses of the vegetation on danish commons. Biol. Skar. 5, 1-34 (1948)

775

60. Crichton, G., Guo, Y., Pyysalo, S., Korhonen, A.: Neural networks for link prediction in realistic biomedical graphs: a multi-dimensional evaluation of graph embedding-based approaches. BMC bioinformatics 19(1), $1-11(2018)$

61. Lu“, L., Zhou, T.: Link prediction in weighted networks: The role of weak ties. EPL (Europhysics Letters) 89(1), 18001 (2010)

62. Chen, H., Zhang, Z., Zhang, J.: In silico drug repositioning based on the integration of chemical, genomic and pharmacological spaces. BMC bioinformatics 22(1), 1-12 (2021)

63. Wang, C., Satuluri, V., Parthasarathy, S.: Local probabilistic models for link prediction. In: Seventh IEEE International Conference on Data Mining (ICDM 2007), pp. 322-331 (2007). IEEE

783

784

64. O’Madadhain, J., Hutchins, J., Smyth, P.: Prediction and ranking algorithms for event-based network data. ACM SIGKDD explorations newsletter 7(2), 23-30 (2005)

785

786

787

65. Backstrom, L., Leskovec, J.: Supervised random walks: predicting and recommending links in social networks. In: Proceedings of the Fourth ACM International Conference on Web Search and Data Mining, pp. 635-644 (2011)

788

789

66. Dong, Y., Tang, J., Wu, S., Tian, J., Chawla, N.V., Rao, J., Cao, H.: Link prediction and recommendation across heterogeneous social networks. In: 2012 IEEE 12th International Conference on Data Mining, pp. 181-190 (2012). IEEE

67. Kerrache, S., Alharbi, R., Benhidour, H.: A scalable similarity-popularity link prediction method. Scientific reports 10(1), 1-14 (2020)

68. Muscoloni, A., Michieli, U., Cannistraci, C.V.: Local-ring network automata and the impact of hyperbolic geometry in complex network link-prediction. arXiv preprint arXiv:1707.09496 (2017)

69. Garcia-Gasulla, D., Ayguad'e, E., Labarta, J., Cort'es, U.: Limitations and alternatives for the evaluation of large-scale link prediction. arXiv preprint arXiv:1611.00547 (2016) 
70. Wang, W., Cai, F., Jiao, P., Pan, L.: A perturbation-based framework for link prediction via non-negative matrix factorization. Scientific reports 6(1), 1-11 (2016)

71. Davis, J., Goadrich, M.: The relationship between precision-recall and roc curves. In: Proceedings of the 23rd International Conference on Machine Learning, pp. 233-240 (2006)

72. Kumar, N.G., Contaifer, D., Madurantakam, P., Carbone, S., Price, E.T., Van Tassell, B., Brophy, D.F., Wijesinghe, D.S.: Dietary bioactive fatty acids as modulators of immune function: implications on human health. Nutrients 11(12), 2974 (2019)

73. do Vale, G.T., Ceron, C.S., Gonzaga, N.A., Simplicio, J.A., Padovan, J.C.: Three generations of $\beta$-blockers: history, class differences and clinical applicability. Current hypertension reviews 15(1), 22-31 (2019)

74. Tritsch, N.X., Granger, A.J., Sabatini, B.L.: Mechanisms and functions of gaba co-release. Nature Reviews Neuroscience 17(3), 139-145 (2016)

75. Jorgensen, E.M.: Gaba. WormBook: The Online Review of C. elegans Biology [Internet] (2005)

76. Ricciotti, E., FitzGerald, G.A.: Prostaglandins and inflammation. Arteriosclerosis, thrombosis, and vascular biology 31(5), 986-1000 (2011)

77. Engelking, L.R.: Textbook of Veterinary Physiological Chemistry, Updated 2/e. Academic Press, (2010)

78. Di Pasquale, E., Chahinian, H., Sanchez, P., Fantini, J.: The insertion and transport of anandamide in synthetic lipid membranes are both cholesterol-dependent. PLoS One 4(3), 4989 (2009)

79. Higgins, A., Lees, P.: The acute inflammatory process, arachidonic acid metabolism and the mode of action of anti-inflammatory drugs. Equine Veterinary Journal 16(3), 163-175 (1984)

80. Delves, P.J., Roitt, I.M.: Encyclopedia of Immunology, pp. 2024-2027. Academic Press, (1998)

81. Baker, R.R.: The eicosanoids: a historical overview. Clinical biochemistry 23(5), 455-458 (1990)

82. Varga, T., Czimmerer, Z., Nagy, L.: Ppars are a unique set of fatty acid regulated transcription factors controlling both lipid metabolism and inflammation. Biochimica et Biophysica Acta (BBA)-Molecular Basis of Disease 1812(8), 1007-1022 (2011)

83. Kaur, N., Chugh, V., Gupta, A.K.: Essential fatty acids as functional components of foods-a review. Journal of food science and technology 51(10), 2289-2303 (2014)

84. Ortuño Sahagu'n, D., M'arquez-Aguirre, A., Quintero-Fabi'an, S., L’opez-Roa, R., Rojas-Mayorqu' in, A.: Modulation of ppar- $\gamma$ by nutraceutics as complementary treatment for obesity-related disorders and inflammatory diseases. PPAR research 2012 (2012)

85. Wu, H.-T., Chen, W., Cheng, K.-C., Ku, P.-M., Yeh, C.-H., Cheng, J.-T.: Oleic acid activates peroxisome proliferator-activated receptor $\delta$ to compensate insulin resistance in steatotic cells. The Journal of nutritional biochemistry 23(10), 1264-1270 (2012)

86. Santos, M.R., Moreira, F.V., Fraga, B.P., Souza, D.P.d., Bonjardim, L.R., Quintans-Junior, L.J.: Cardiovascular effects of monoterpenes: a review. Revista Brasileira de Farmacognosia 21(4), 764-771 (2011) 
87. Peixoto-Neves, D., Wang, Q., Leal-Cardoso, J.H., Rossoni, L.V., Jaggar, J.H.: Eugenol dilates mesenteric arteries and reduces systemic bp by activating endothelial cell trpv 4 channels. British journal of pharmacology 172(14), 3484-3494 (2015)

88. Farzam, K., jan, A.: Beta blockers. StatPearls (2021)

89. Alhayek, S., Preuss, C.V.: Beta 1 receptors. StatPearls (2021)

90. Remiszewska, M., Jastrzebski, Z., Czyzewska-Szafran, H., Wutkiewicz, M.: Antihypertensive treatment with beta blockers and gabaergic transmission in rat brain. Acta Poloniae Pharmaceutica 52(2), 185-186 (1994)

91. Bernal-Morales, B., Cueto-Escobedo, J., Guill'en-Ruiz, G., Rodr'1guez-Landa, J.F., Contreras, C.M.: A fatty

\section{Supplementary Information}

Additional file 1: Figure S1. The DrugBank Dataset extraction procedure. Figure S2. The FooDB dataset extraction procedure. Figure S3. Calculating Structure Similarity Profile Using Tanimoto Coefficient. Table S1. Calculating the contribution of a food compound in a food. Figure S4. Disjoint and Joint Graph. Figure S5. Precision@Top. Figure S6. Precision@top comparison of eight different methods over the disjoint graph network. Figure S7. Precision@top comparison of eight different methods over the joint graph network. Figure S8. Area Under the Curve (AUC) for path category-based (dataset 1: disjoint graph). Figure S9. Area Under the Curve (AUC) for path category-based (dataset 2: joint graph). Figure S10. Area Under the Curve (AUC) for neighborhood-based similarity-based (dataset 1: disjoint graph). Figure S11. Area Under the Curve (AUC) for neighborhood-based similarity-based (dataset 2: joint graph). Figure S12. Precision-Recall Curve (PRC) for path category-based (dataset 1: disjoint graph). Figure S13. PrecisionRecall Curve (PRC) for path category-based (dataset 2: joint graph). Figure S14. Precision-Recall Curve (PRC) for path neighborhood-based similarity-based (dataset 1: disjoint graph). Figure S15. Precision-Recall Curve (PRC) for neighborhood-based similarity-based (dataset 2: joint graph). Table S2. Number of links in the graph after applying different food compound contribution score.

Additional file 2: Table S1. Top 10 FDIs found from path category-based (path length-2) method over disjoint graph. Records might appear repeated, but food item IDs are different in this table. Table S2. Top 20 FDIs from path category-based (path length-2) method over disjoint and joint graph. Table S3. Top 10 FDIs from Neighborhood-based 863 Similarity-based method over joint graph.

864 Additional file 3: Table S1. Top 25 FDIs found from path category-based (path length-2) method over disjoint graph. 865 Table S2. Top 25 FDIs found from path category-based (path length-2) method over joint graph. Table S3. Top 20 
866 common FDIs found from path category-based (path length-2) method over disjoint and joint graph. Table S4. Top 86725 FDIs from Neighborhood-based similarity-based methods method over the joint graph. 


\section{Supplementary Files}

This is a list of supplementary files associated with this preprint. Click to download.

- AdditionalFile1.docx

- AdditionalFile2.docx

- AdditionalFile3.docx 


\section{Supplementary Files}

This is a list of supplementary files associated with this preprint. Click to download.

- AdditionalFile1.docx

- AdditionalFile2.docx

- AdditionalFile3.docx 